\title{
Uncovering the Immunological Properties of Isolated Lymphoid Follicles
}

\author{
Janice Layhadi ${ }^{1}$ and Mohamed Shamji ${ }^{1}$ \\ ${ }^{1}$ Imperial College London
}

July 22, 2020

Article type: News and Views: Groundbreaking Discoveries in Immunology

Title: Uncovering the Immunological Properties of Isolated Lymphoid Follicles

Authors: Janice A. Layhadi ${ }^{1}$ and Mohamed H. Shamji ${ }^{1}$

${ }^{1}$ Immunomodulation and Tolerance Group, Allergy and Clinical Immunology, National Heart and Lung Institute, Imperial College London, UK

Correspondence: Janice Layhadi, Immunomodulation and Tolerance Group, Allergy and Clinical Immunology, National Heart and Lung Institute, Imperial College London, UK

Email address: Janice.lee-layhadi07@imperial.ac.uk

Keywords: ILF; GALT; secretory IgA; intestine; adaptive immunity

Abbreviations: CyTOF, Mass Cytometry; GALT, Gut-Associated Lymphoid Tissue; GC, Germinal Centre; ILF, Isolated Lymphoid Follicles; PP, Peyer's patch; sIgA, secretory IgA; Tfh, T follicular helper cells; Th, T helper cells;Treg, T regulatory cells

Acknowledgement: We would like to thank Anna Globinska for her assistance in generating the graphical figure.

\section{Main text}

The intestine is the largest organ of the body and exhibits the greatest amount and diversity of immune cells. Intestinal homeostasis is tightly controlled by the gut mucosal immune network, which involves gutassociated lymphoid tissues (GALT), secretory IgA (sIgA), mucosal immune cells (i.e. T helper (Th), T follicular (Tfh) and T regulatory (Treg) cells), cytokines, chemokines and commensal bacteria, ${ }^{1}$ among others. Unsurprisingly, crosstalk between these cells and their local environment is crucial for the maturation and function of the immune system, and the disruption of this communication often leads to the development of immune disorders ${ }^{2}$.

GALT comprises of the Peyer's Patches (PP) as well as isolated lymphoid follicles (ILFs), which are present throughout the human intestine. The role of GALT in adaptive immune responses within the intestine has been primarily reported in mice, with a recent report revealing a role for PP in supplying IgA ${ }^{+}$plasmablasts to the small intestine. ${ }^{3}$ However, functional studies of human GALT have been hampered due to the scarcity of established protocols for isolation of pure cells and immunological profiling.

Fenton et al. describe a novel method for isolating human GALT that allows thorough profiling of immune cells in ILFs. ${ }^{4}$ The conventional isolation method has identified the existence of plasma cells, naïve B and $\mathrm{T}$ cells in colonic cell suspensions. The novel isolation method proposed by Fenton et al . revealed that naïve lymphocytes and plasma cells were restricted to ILFs and to GALT-free lamina propria (LP), respectively. 
This cellular distribution indicates that ILFs (both mucosal and sub-mucosal) function as inductive sites of the adaptive immune response. ${ }^{4}$ Using mass cytometry, CyTOF, they also demonstrated that the composition of T and B cells in the GALT was distinct from the GALT-free LP; CD57 ${ }^{\text {hi }} \mathrm{PD}-1^{\text {hi }}$ Tfh-like cells and $\mathrm{CD} 4^{+} \mathrm{CD} 25^{+} \mathrm{CD} 127^{-} \mathrm{IL}-2^{-}$Treg cells primarily localized in the GALT while cytokine-producing $\mathrm{T}$ cell subsets mostly localized within the GALT-free LP. ${ }^{4}$ Moreover, GALT ILFs comprise of germinal centre (GC) and memory B cells that express IgM, IgG, IgA1 and IgA2, which indicates that B cell class switching occurs within the GC in these cell compartments and is a source of intestinal $\operatorname{IgA}$. IgA sequencing analysis revealed minimal overlap in the IgA repertoire of GALT follicles. A low overlap of IgA clones was observed between the PP and sub-mucosal ILFs suggesting a distinct profile within the intestinal immune response. When the most abundant clones of the sub-mucosal ILFs were further investigated, they identified that these clones made up a huge fraction of the total clonal repertoire of the colon LP. This suggests that sub-mucosal ILFs serve as important adaptive-inductive sites in the colon.

The study by Fenton et al . advances the understanding of ILFs in initiating the adaptive immune response and generating intestinal IgA, especially in the context of allergic diseases. ILFs are commonly seen on intestinal biopsies of young children, and hypertrophy of these follicles has been associated with allergic sensitization. ${ }^{5}$ Moreover, the work by Fenton et al. showing ILFs as a key source of intestinal IgA highlights their potential role in the induction of immune tolerance and as a therapeutic target. sIgA functions as a defence system against pathogen and toxins and, its reduction has been associated with increased risk of allergy development. This is supported by studies showing that low salivary and intestinal sIgA levels resulted in an increased risk of allergic manifestations during childhood. ${ }^{6}$ Allergen sensitization studies in mice using bovine lactoglobulin also demonstrated suppression in intestinal allergen-specific sIgA and number of $\operatorname{Ig} \mathrm{A}^{+}$ $\mathrm{B}$ cells in PP compared with tolerant mice. ${ }^{7}$ However, a recent clinical study showed contradicting findings whereby a weak correlation was observed between IgA deficiency and food allergy. ${ }^{8}$

The interaction between sIgA and commensal bacteria in the intestine is poorly understood. Children developing allergic manifestations such as asthma have shown a lower proportion of IgA bound to faecal bacteria compared to healthy children. This raises the possibility that sIgA shapes the gut microbiota for inducing oral tolerance, in addition to its role in maintaining homeostasis towards gut commensal bacteria. Finally, as ILFs harbour GCs, identifying their role in allergic diseases would be highly relevant because $\mathrm{T}$ and $\mathrm{B}$ cell interaction at the GC is known to generate high-affinity, inflammatory antibodies (IgE). ${ }^{9}$

These novel findings show the role of human ILFs as a primary core for regional immunity in the human intestine. Moreover, this study paves the way towards a better understanding of the mechanisms involved in maintaining the intestinal homeostatic network, allowing the development of preventive and therapeutic approaches to immune disorders, including allergic diseases.

\section{Total Word count: 747}

Figure legend

Figure 1. Role of ILF within the GALT of the intestine. ILF consist of a heterogeneous population of immune cells that includes DCs, T cells, Tfh-like cells and Treg cells. Moreover, they comprise of B cells, IgA + B cells, IgG+ B cells and IgM+ B cells, supporting their role in the class switching of B cells. Human ILFs plays a role as crucial immune inducer sites of adaptive immune response with an ability to produce secretory IgA that plays a role in providing the first line of defence in the intestinal tissue.

DCs, dendritic cells; Ig, Immunoglobulin; ILF, isolated lymphoid follicles; Tfh, T follicular helper cells.

\section{References}

1. Tokuhara, D., et al. A comprehensive understanding of the gut mucosal immune system in allergic inflammation. Allergol Int68 , 17-25 (2019).

2. Durack, J. \& Lynch, S.V. The gut microbiome: Relationships with disease and opportunities for therapy. J Exp Med 216 , 20-40 (2019). 
3. Masahata, K., et al. Generation of colonic IgA-secreting cells in the caecal patch. Nat Commun $\mathbf{5}, 3704$ (2014).

4. Fenton, T.M., et al. Immune Profiling of Human Gut-Associated Lymphoid Tissue Identifies a Role for Isolated Lymphoid Follicles in Priming of Region-Specific Immunity. Immunity 52 , 557-570 e556 (2020).

5. Kokkonen, J. \& Karttunen, T.J. Lymphonodular hyperplasia on the mucosa of the lower gastrointestinal tract in children: an indication of enhanced immune response? J Pediatr Gastroenterol Nutr 34 , 42-46 (2002).

6. Kukkonen, K., et al. High intestinal IgA associates with reduced risk of IgE-associated allergic diseases. Pediatr Allergy Immunol 21, 67-73 (2010).

7. Frossard, C.P., Hauser, C. \& Eigenmann, P.A. Antigen-specific secretory IgA antibodies in the gut are decreased in a mouse model of food allergy. J Allergy Clin Immunol 114, 377-382 (2004).

8. Aghamohammadi, A., et al. IgA deficiency: correlation between clinical and immunological phenotypes. J Clin Immunol29 , 130-136 (2009).

9. Gould, H.J., Takhar, P., Harries, H.E., Durham, S.R. \& Corrigan, C.J. Germinal-centre reactions in allergic inflammation. Trends Immunol 27 , 446-452 (2006).

\section{Hosted file}

Figure 1.docx available at https://authorea.com/users/345057/articles/471416-uncovering-theimmunological-properties-of-isolated-lymphoid-follicles 